\title{
$Q$ fever in the Netherlands: an update on the epidemiology and control measures
}

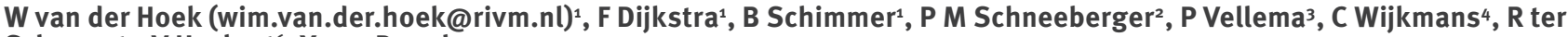

Schegget ${ }^{5}$, V Hackert ${ }^{6}$, Y van Duynhoven ${ }^{1}$

1. Centre for Infectious Disease Control, National Institute for Public Health and the Environment (Rijksinstituut voor

Volksgezondheid en Milieu, RIVM), Bilthoven, the Netherlands

2. Jeroen Bosch Hospital, 's-Hertogenbosch, the Netherlands

3. Animal Health Service, Deventer, the Netherlands

4. Municipal Health Service "Hart voor Brabant",'s-Hertogenbosch, the Netherlands

5. Municipal Health Service Brabant-Southeast, Eindhoven, the Netherlands

6. Municipal Health Service South Limburg, Sittard-Geleen, the Netherlands

\section{Citation style for this article:}

Citation style for this article: van der Hoek W, Dijkstra F, Schimmer B, Schneeberger PM, Vellema P, Wijkmans C, ter Schegget R, Hackert V, van Duynhoven Y. Q fever in the Netherlands: an update on the epidemiology and control measures. Euro Surveill. 2010;15(12):pii=19520. Available online: http://www.eurosurveillance.org/ ViewArticle.aspx?Articleld $=19520$

This article has been published on 25 March 2010

Since the steady rise in human cases which started in 2007, Q fever has become a major public health problem in the Netherlands with 2,357 human cases notified in the year 2009. Ongoing research confirms that abortion waves on dairy goat farms are the primary source of infection for humans, primarily affecting people living close (under $5 \mathrm{~km}$ ) to such a dairy goat farm. To reverse the trend of the last three years, drastic measures have been implemented, including the large-scale culling of pregnant goats on infected farms.

\section{Extent of the problem}

The Netherlands is facing a $Q$ fever problem that is still increasing in size since the last report in this journal [1]. In 2009, a total of 2,357 new Q fever patients were registered in the national infectious disease notification database. These patients fulfilled the case notification criteria of fever, pneumonia, or hepatitis, combined with a positive laboratory result. $Q$ fever infection is asymptomatic in $60 \%$ of cases [2] and if symptomatic often presents as influenza-like illness. Increasing awareness among patients and doctors will likely result in detection of more relatively mild cases. However, the proportion of notified patients that had to be admitted to a hospital seems to have stabilised around $20 \%$, after a decline from 2007 to 2008 (Table).
The high percentage of $50 \%$ in 2007 was largely influenced by active case finding in a retrospective survey among hospitalised cases. The $19.7 \%$ of 2009 are still much higher than the $2-5 \%$ hospitalisation that are reported in the literature [2].

In the first 10 weeks of 2010 a considerable number of cases has already been notified, which, based on experiences of preceding years and taking into account the drastic measures taken, was not expected at this time of year (Figure 1).

The intensified media attention in late 2009 and early 2010 might have caused an increase in the number of consultations. Improved laboratory capacity will also have influenced the number of notified cases and quality of the notified data. As can be seen in the epidemiological curve of $\mathbf{2 0 0 7}$ there is a more or less scattered pattern due to delayed notifications. From 2009 onwards, routine PCR is included in the diagnostic workup of acute $Q$ fever, which can accelerate diagnosis. However, PCR is only positive in early $Q$ fever. The high background of notified cases in the winter of 2009-10 may be a reflection of high seroprevalence among the affected population, due to persisting antibodies, both IgG and IgM phase II, used to diagnose acute $\mathrm{Q}$-fever. This, combined with non-specific clinical

TABLE

Hospital admissions for Q fever by year of notification, the Netherlands 2007-2009

\begin{tabular}{|l|c|c|c|c|c|c|}
\hline Year of notification & Notified cases & \multicolumn{3}{|c|}{ Admitted to hospital } & \multicolumn{2}{c|}{ Percentage admitted (95\% CI) } \\
\hline & & Yes & No & Information missing & & \\
\hline 2009 & 2,357 & 459 & 1,869 & 29 & $19.7 \%$ & $(18.1-21.3)$ \\
\hline 2008 & 1,000 & 207 & 785 & 8 & $20.9 \%$ & $(18.4-23.5)$ \\
\hline 2007 & 168 & 83 & 83 & 2 & $50.0 \%$ & $(42.4-57.6)$ \\
\hline
\end{tabular}

$\mathrm{Cl}$ : confidence interval. 
symptoms of $Q$ fever, makes it difficult to differentiate between acute and past $Q$ fever infections, and thus makes notifications less accurate.

In 2009, six deaths were reported, all in patients with other underlying medical conditions. The median age of the patients was 49 (interquartile range (IQR): 38-59), and 61\% were male. In 2008, the median age was 50 years (IQR: 41-59), with 64\%, male cases. Geographically, the epidemic affected an area that was larger than in the preceding years. A large new cluster was observed in Limburg, the southernmost province of the Netherlands, near a Q fever-affected dairy goat farm functioning as a healthcare farm (daily activities).

There is consensus among public health and veterinary professionals that most of the human $Q$ fever cases are linked to abortion waves on large dairy goat farms, and to a much lesser extent on dairy sheep farms. Consequently, interventions have focused on these types of farms. However, there are indications that direct contact with non-dairy sheep has also caused a limited number of human cases: at least 28 among patients and staff of a mental health institution [3] and possibly up to 46 among 12,000 people who visited a sheep farm during the lambing season in February and March 2009.

\section{Control measures}

In February 2009, a nationwide hygiene protocol became mandatory for professional dairy goat and dairy sheep farms. Between April and November 2009, approximately 250,000 small ruminants were mandatorily vaccinated, including those on farms in the highincidence area in the south of the country (Figure 2), farms with a recent history of $Q$ fever, and farms offering recreational activities. Veterinarians, physicians and the public were informed through targeted mailings, publications and the media. On 1 October 2009, bulk milk monitoring became mandatory on farms with more than 50 dairy goats or dairy sheep, and PCRpositive bulk milk has since been used as an additional criterion for veterinary notification of $Q$ fever. The initial frequency of testing each farm every other month has been increased to once every two weeks as of 14 December 2009. By 18 February 2010, 74 dairy goat farms and two dairy sheep farms, out of the total of 360 dairy goat farms and 40 dairy sheep farms with more than 50 animals in the Netherlands, had been declared Q fever-infected based on PCR-positive bulk milk testing (Figure 2). The number of positive farms is expected to increase further towards the peak of the lambing season (March-April).

In retrospect, a large human cluster in an urban area in 2008 could clearly be linked to a dairy goat farm (with over 400 animals) with a $Q$ fever related abortion wave a few weeks before the first human cases presented

\section{FIGURE 1}

$\mathrm{Q}$ fever notifications by year and week

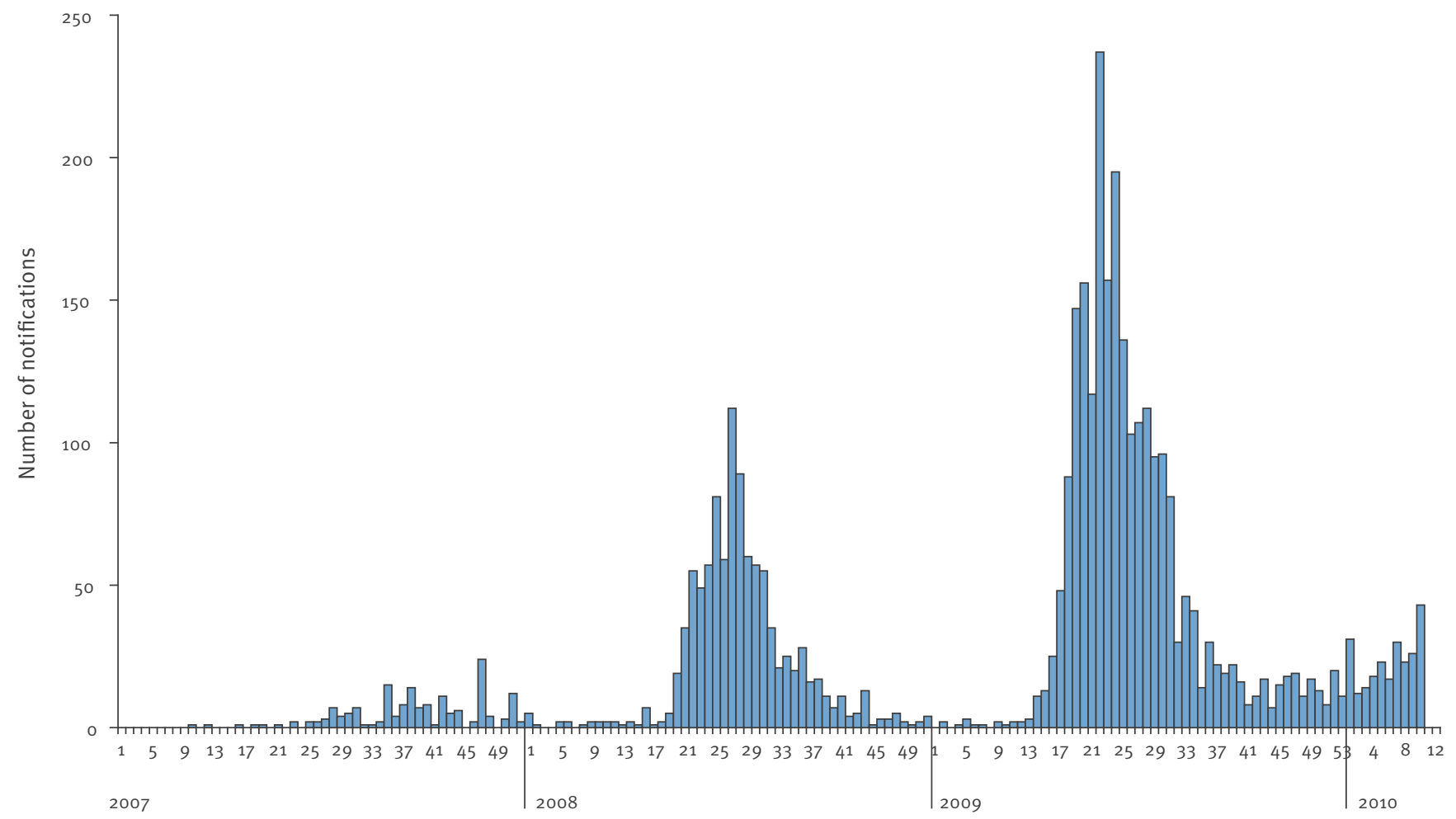

Year and week of notification 
[4]. People living within $2 \mathrm{~km}$ of the farm had a much higher risk for $Q$ fever than those living more than 5 $\mathrm{km}$ away (relative risk 31.1; 95\% confidence interval (CI): 16.4-59.1). Based on this study, a public information campaign has been targeted to zones of increased risk around affected farms. When a dairy goat or dairy sheep farm tests positive for Coxiella burnetii in a bulk milk sample for the first time, all inhabitants living within a radius of $5 \mathrm{~km}$ of the farm receive a letter informing them on the presence of a $Q$ fever-positive farm in their proximity. The letter gives no specific advice, but allows people with known risk factors to avoid the farm. The list of positive farms is updated daily and is available to the public on the website of the Food and Consumer Product Safety Authority (www. vwa.nl, in Dutch).

In $2009,59 \%$ of the notified human Q fever cases lived within a radius of $5 \mathrm{~km}$ of an infected dairy goat or dairy sheep farm, while only $12 \%$ of the Dutch population live within these zones. The incidence of $Q$ fever

\section{FIGURE 2}

Incidence of human $\mathrm{Q}$ fever by municipality $(\mathrm{n}=2,357)$ and locations of $\mathrm{Q}$ fever infected dairy goat and dairy sheep farms, the Netherlands, 2009

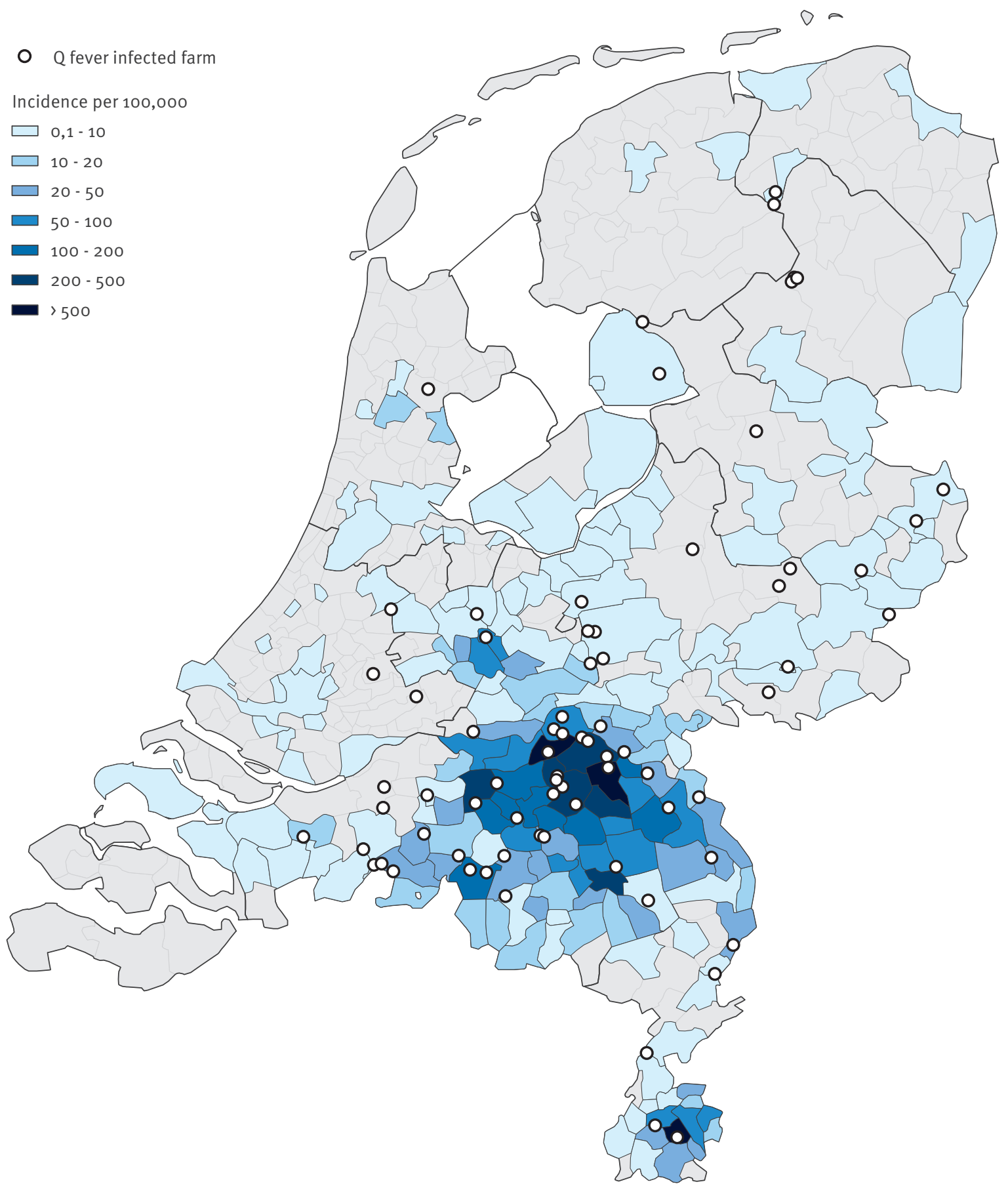

Map compiled by Ben Bom, Expertise Centre for Methodology and Information Services, RIVM. 
in 2009 was 69 per 100,000 population within, and six per 100,000 outside the $5 \mathrm{~km}$-areas.

\section{Screening of pregnant women}

International literature suggests that a $Q$ fever infection during pregnancy may lead to adverse pregnancy outcome in a large percentage of cases [5]. However, in a recently completed retrospective study in the high incidence area in the Netherlands, the presence of antibodies against $C$. burnetii in early pregnancy was not significantly associated with preterm delivery, low birth weight, or perinatal mortality (van der Hoek et al., unpublished data). A large-scale prospective screening and treatment study coordinated by the University Medical Centre Groningen, was started in March 2010, aimed at providing more conclusive data on the need to screen pregnant women in high incidence areas.

\section{Screening of blood donors}

There is a theoretical risk for transmission of $C$. burnetii through blood transfusion. Preliminary results indicate that in 2009, C. burnetii DNA was detected in a small minority of blood donations in the affected area. The risk of infection is probably negligible, but as a precautionary measure, Sanquin Blood Supply Foundation, the organisation responsible for all blood products in the Netherlands, started screening donated blood for C. burnetii in the high-incidence area of the country on 15 March 2010.

\section{Expectations for 2010}

The veterinary interventions, especially vaccination, animal movement restrictions, culling and hygiene measures, are expected to have an impact in 2010 and 2011. However, the resilience of $C$. burnetii in the environment and the possible role of animal species other than small ruminants make a prediction difficult but could potentially lead to incidence levels not much lower or even higher than those observed in 2009. If spores persist in stables even after removing all animals or if environmental contamination and different animal reservoirs turn out to become relatively more important for transmission to humans, the striking seasonal pattern of the years 2007-2009 may be altered, possibly resulting in a more erratic transmission pattern over time. So far, there are no signs that the $\mathrm{Q}$ fever problem is spreading to neighbouring countries. It could be that factors such as lower population density, lower animal density, and different animal production methods in Belgium and Germany, compared to the Netherlands, play a role.

\section{Research agenda}

To fill the many knowledge gaps regarding $Q$ fever, there is a large interdisciplinary research agenda in the Netherlands focusing on human and veterinary public health and individual patient care. Further elucidating the source and transmission routes will to a large extent depend on advances in molecular biology. Conclusive matching of the bacteria that are found in animals with human and environmental samples has not yet been successful. Obtaining $C$. burnetii DNA is difficult, except from placenta material of infected goats and sheep. Typing by multiple-locus variablenumber tandem repeat analysis (MLVA) has been used on a limited scale in human and veterinary samples and indicated similarity in strains isolated from a small sheep herd with strains from the human cluster in the mental health institution in May 2008 [6]. However, while various different MLVA types were identified, research by the Central Veterinary Institute in Lelystad shows that one MLVA type prevails on many dairy goat farms in the high risk area in the southern part of the Netherlands, possibly indicating clonal spread in this area [7]. This hampers tracing the source of human $Q$ fever in the high-risk area to a specific farm. In a newly started project, whole genome sequencing will be used to be able to distinguish between Coxiella bacteria from different sources.

\section{Discussion}

$\mathrm{Q}$ fever is now considered a major public health problem in the Netherlands and has recently led to drastic measures, including the large-scale culling of pregnant goats and sheep. Despite the strictest veterinary measures possible, $Q$ fever is expected to remain a significant problem over the coming years. The control measures are aimed at stabilising the number of human cases in 2010, while the sustained compulsory vaccination campaign in small ruminants which is implemented in 2010 nationwide for target farms, is expected to eventually cause a drop in human cases in 2011 and subsequent years.

\section{References}

1. Schimmer B, Dijkstra F, Vellema P, Schneeberger PM, Hackert $\mathrm{V}$, ter Schegget $\mathrm{R}$, et al. Sustained intensive transmission of $\mathrm{Q}$ fever in the south of the Netherlands, 2009. Euro Surveill. 2009;14(19). pii=19210. Available from: http://www. eurosurveillance.org/ViewArticle.aspx?Articleld=19210

2. Raoult D, Marrie TJ, Mege JL. Natural history and pathophysiology of Q fever. Lancet Infect Dis. 2005;5(4):219-26.

3. Koene RPM, Schimmer B, Rensen H, Biesheuvel M, de Bruin $A$, Lohuis A, et al. A Q fever outbreak in a psychiatric care institution in the Netherlands. Epidemiol Infect. 2010 Feb 9:16. doi:10.1017/S095026881000021X.

4. Schimmer B, ter Schegget R, Wegdam M, Züchner L, de Bruin $A$, Schneeberger $P$, et al. The use of a geographic information system to identify a dairy goat farm as the most likely source of an urban Q-fever outbreak. BMC Infect Dis. 2010;10(1):69. doi: $10.1186 / 1471-2334-10-69$.

5. Carcopino X, Raoult D, Bretelle F, Boubli L, Stein A. Q Fever during pregnancy: a cause of poor fetal and maternal outcome. Ann N Y Acad Sci. 2009;1166:79-89.

6. Klaassen CHW, Nabuurs-Franssen MH, Tilburg JJHC, Hamans MAWM, Horrevorts AM. Multigenotype $Q$ fever outbreak, the Netherlands [letter]. Emerg Infect Dis. 2009;15(4):613-4.

7. Roest H-J. Geographical distribution of MLVA types of Coxiella burnetii isolates from goats in The Netherlands. Annual Scientific Meeting, Med-Vet-Net Association, 3-6 June 2009, Madrid, Spain. 